\title{
A unique case report of 2-cm-long fishbone induced acute suppurative thyroiditis
}

\author{
Yun Gao ${ }^{2}$, Kan Chen ${ }^{2}$, Likun Yang ${ }^{1}$, Yi Feng' ${ }^{1}$ Jie Zhu ${ }^{1}$, Yuhai Wang ${ }^{1}$, Xing Lin Feng ${ }^{3 *}$ and Wei Lin ${ }^{1,2 *}$ \\ ${ }^{1}$ Department of neurosurgery, No 101 Hospital of Chinese PLA, China \\ ${ }^{2}$ JiangYuan Hospital Affiliated to Jiangsu Institute of Nuclear Medicine, China \\ ${ }^{3}$ Department of Health Policy and Administration, School of Public Health, Peking University, China
}

\begin{abstract}
Acute suppurative thyroiditis (AST) is an infectious disease of the thyroid gland caused by bacterial, fungal, and parasitic organisms. Its diagnosis is often delayed, which may lead to life-threatening hazards. This study presents a unique case of foreign matter induced AST who presented as a normal AST case in an adult female. After the antibiotics treatment, when the pain symptom released and the vital signs were normal, surprisingly, CT scan and ultrasound revealed a foreign matter in the patient's thyroid, and thus an operation was conducted. A 2 -cm-long fishbone was discovered in the thyroid tissue. This particular case was unique due to fact that the infection was induced by a fishbone piercing across the thyroid gland. Since thyroidectomy should be promptly performed for such a case, the consideration of foreign mattes induced AST should be borne in mind for discrimination diagnosis.
\end{abstract}

\section{Introduction}

Acute suppurative thyroiditis (AST) is a rare and potentially lifethreatening endocrine emergency. The incidence of AST accounts for $0.1 \%-0.7 \%$ of all thyroid diseases [1]. AST is a relatively rare clinical condition because the thyroid gland is remarkably resistant to infections due to its high iodine content, hydrogen peroxide production, blood supply network, abundant lymphatic drainage, and encapsulated location. A well-known causal association is congenital abnormality of the piriform sinus, underlying autoimmune diseases, or an otherwise immunocompromised host. However, unlike the preceding studies, the present paper reports a unique case of AST caused by a fishbone just in the infective region of the thyroid which has not been reported in surgery settings [2].

\section{Case report}

A 41-year-old Chinese woman complained of left-anterior neck pain for 6 months before her first visit to the doctor. Subacute thyroiditis in the local hospital and was treated with glucocorticoids. However, after drug withdrawal for 1 month, neck pain emerged again, and glucocorticoid was used again. So that the patient was transferred to the Jiangyuan Hospital, Jiangsu, China, after her symptoms worsened. She was a nonsmoker and nondrinker. However, she was allergic to penicillin. The patient complained to the doctor: about more than 6 months ago, she felt pain in the throat and neck after eating fishes. Additional medical history was unremarkable.

Her vital signs at the time of admission to the hospital ward were as follows: blood pressure $120 / 70 \mathrm{mmHg}$, pulse rate $71 / \mathrm{min}$, respiratory rate $18 / \mathrm{min}$, and temperature $36.5^{\circ} \mathrm{C}$. Laboratory tests showed a leukocyte count of $9790 / \mathrm{mL}$, an erythrocyte sedimentation rate of $10 \mathrm{~mm} / \mathrm{h}$, a free thyroxine (T4) level of $17.87 \mathrm{pmol} / \mathrm{L}$ (normal range, 11.46-23.17), a free tri-iodothyronine (T3) level of $5.51 \mathrm{pmol} / \mathrm{L}$ (normal range, 2.80-7.10), a thyrotropin (also known as thyroidstimulating hormone or TSH) level of $2.170 \mathrm{mU} / \mathrm{mL}$ (normal range,
0.35-5.50), and anti-thyroid peroxidase level of $41.44 \mathrm{IU} / \mathrm{mL}$ (normal range, $0-35$ ), and anti-thyroglobulin antibody and anti-TSH receptor antibody (TSH binding inhibitory immunoglobulin) were negative.

On the following day, thyroid ultrasonography was arranged, which revealed a hypoechoic lesion over the left lobe. This sign and laboratory results all supported clinical diagnosis of AST. A computed tomography (CT) scan of the cervical spine revealed no anatomic abnormality in the pharynx. Culture results showed Streptococcus and bacteroides; therefore, the patient was treated with cephalosporin antibiotics. Fine needle aspiration was performed and pus-like fluids were collected. And then, antibiotic was used to treat the suppurative thyroiditis from February 28 until March 28 . When the pain symptom released, the patient was reexamined by CT scan and ultrasound. A neck reconstructive CT scan showed an abscess with air collection in the left thyroid lobe (Figure 1). Surprisingly, a bone-like foreign matter was discovered in the right place where the thyroid was affected by acute suppurative thyroiditis. So that when the pain symptom subsided and the vital signs were normal, the patient underwent partial thyroidectomy after 28 days' admission. Histologic studies of the resected thyroid revealed a large area of interstitial fibrosis with small follicles or solid clusters of follicular cells without colloid, indicating previous severe inflammation. Finally, a 2-cm-long fishbone was discovered after thyroidectomy finally (Figure 2).

Correspondence to: Wei Lin, Department of neurosurgery, No 101 Hospital of Chinese PLA, Wuxi 214044, China, E-mail: smithlinwx@aliyun.com

Xing Lin Feng, Department of Health Policy and Administration, School of Public Health, Peking University, Beijing, China, E-mail: fxl@bjmu.edu.cn

Key words: acute suppurative thyroiditis, fish bone, case report, thyroidectomy, life-threatening

Received: August 08, 2016; Accepted: August 29, 2016; Published: August 31, 2016 

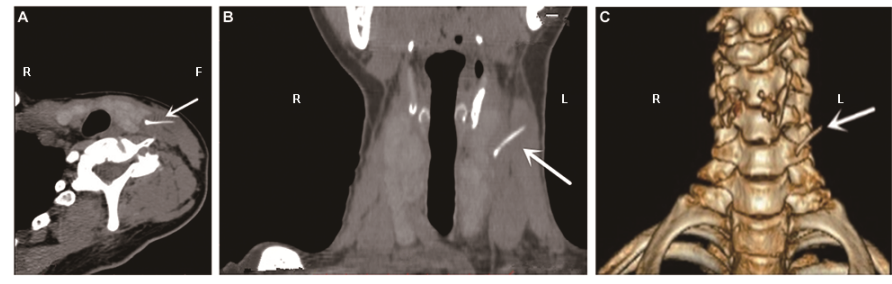

Figure 1. Neck CT reconstruction of fishbone from different angles (white arrow).

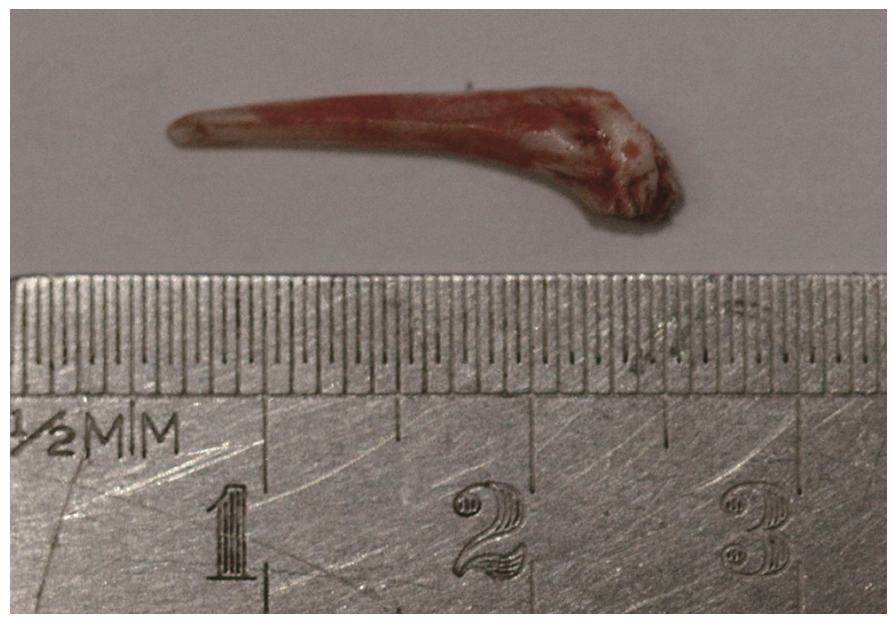

Figure 2. A 2-cm-long fishbone.

\section{Discussion}

Infectious acute thyroiditis is a life-threatening disorder and the prognosis depends on prompt recognition and treatment. Bacterial, fungal, and even parasitic organisms have been documented as etiologic agents [3-6]. In the present case, the responsible pathogens were Streptococcus and bactericides based on culture results. This particular case was unique because the infection was induced by a foreign matterfish bone- piercing across the thyroid gland. This is unique since our systematic search of the Medline just found rare clinical reports for foreign body induced AST, besides one case not identified in surgery settings [2] and the other by a chicken bone [7].

Asian people have the habit of eating fish with many fish bones; therefore, the chances of a fishbone getting stuck are high in the muscle of the pharyngeal-esophagus airway. In such a condition, swallowing other food to push or squeeze the stuck fishbone in the pharyngeal airway or the esophagus may actually usher in the piercing through of the bone to thyroid tissues. This case made it evident that forcing or swallowing food to resist the stuck fishbone can cause the fishbone to go through the smooth muscle of the esophagus by squeezing, which can further pierce into vital tissues such as the soft tissue of the neck and damage the thyroid gland, including the cervical blood vessels, leading to infection and life-threatening consequences.

Since the Asian people have the habit of eating fish with many fish bones, this unique case shed light on for surgeons that work in Asian settings for the discrimination diagnosis of AST.

\section{Grant support or research funding}

This research received no specific grant from any funding agency in the public, commercial, or not-for-profit sectors.

\section{Declaration of conflicting interests}

All authors declare that they have no any conflict of interests.

\section{Acknowledgements}

None.

\section{References}

1. Paes JE, Burman KD, Cohen J, Franklyn J, McHenry CR, et al. (2010) Acute bacterial suppurative thyroiditis: a clinical review and expert opinion. Thyroid 20: 247-255. [Crossref]

2. Tien KJ, Chen TC, Hsieh MC, Hsu SC, Hsiao JY, et al. (2007) Acute suppurative thyroiditis with deep neck infection: a case report. Thyroid 17: 467-469. [Crossref]

3. Fukata S, Miyauchi A, Kuma K, Sugawara M (2002) Acute suppurative thyroiditis caused by an infected piriform sinus fistula with thyrotoxicosis. Thyroid 12: 175-178. [Crossref]

4. Marui S, de Lima Pereira AC, de Araújo Maia RM, Borba EF (2014) Suppurative thyroiditis due to aspergillosis: a case report. J Med Case Rep 8: 379. [Crossref]

5. Wu C, Zhang Y, Gong Y, Hou Y, Li S, et al. (2013) Two cases of bacterial suppurative thyroiditis caused by Streptococcus anginosus. Endocr Pathol 24: 49-53.

6. Aamir S, Rizvi AA (2012) Visual vignette. Suppurative thyroiditis due to Nocardia asteroides. Endocr Pract 18: 426. [Crossref]

7. Yung BC, Loke TK, Fan WC, Chan JC (2000) Acute suppurative thyroiditis due to foreign body-induced retropharyngeal abscess presented as thyrotoxicosis. Clin Nucl Med 25: 249-252. [Crossref]

Copyright: (C2016 Gao Y. This is an open-access article distributed under the terms of the Creative Commons Attribution License, which permits unrestricted use, distribution, and reproduction in any medium, provided the original author and source are credited. 